\title{
Intraoperative Mortality in Malawi
}

\author{
Meghan Prin, MD, MS,* Stephanie Pan, MS, † Janey Phelps, MD, $\neq$ Godfrey Phiri, CO,§ \\ Guohua Li, MD, DrPH,\|T and Anthony Charles, MD, MPH\#
}

\begin{abstract}
BACKGROUND: Surgical care is essential to improving population health, but metrics to monitor and evaluate the continuum of surgical care delivery have rarely been applied in low-resource settings, and improved efforts at benchmarking progress are needed. The objective of this study was to measure the intraoperative mortality at a Central Referral Hospital in Malawi, evaluate whether there have been changes in intraoperative mortality between 2 time periods, and assess factors associated with intraoperative mortality.
\end{abstract}

METHODS: This was a retrospective cohort study of patients undergoing surgery at Kamuzu Central Hospital in Lilongwe, Malawi. Data describing daily consecutive operative cases were collected prospectively during 2 time periods: 2004-2006 (early cohort) and 2015-2016 (late cohort). The primary outcome was intraoperative mortality. Inverse probability of treatment weighting was used to analyze the association of intraoperative mortality with time using logistic regression models. Multivariable logistic models were performed to evaluate factors associated with intraoperative mortality.

RESULTS: There were 21,090 surgeries performed during the 2 time periods, with 15,846 (75\%) and 5244 (25\%) completed from 2004 to 2006 and 2015 to 2016, respectively. Intraoperative mortality in the early cohort was 57 deaths per 100,000 surgeries (95\% confidence interval [Cl], 26-108) and in the late cohort was 133 per 100,000 surgeries (95\% Cl, 56-286), with 76 per 100,000 surgeries ( $95 \% \mathrm{Cl}, 44-124)$ overall. After applying inverse probability of treatment weighting, there was no evidence of an association between time periods and intraoperative mortality (odds ratio [OR], 1.6; 95\% Cl, 0.9-2.8; $P=.08$ ). Factors associated with intraoperative mortality, adjusting for demographics, included American Society of Anesthesiology physical status III or IV versus I or II (OR, 4.4; 95\% Cl, 1.5-12.5; $P=.006)$ and emergency versus elective surgery (OR, 7.7; 95\% Cl, 2.5-23.6; $P<.001)$.

CONCLUSIONS: Intraoperative mortality in the study hospital in Malawi is high and has not improved over time. These data demonstrate an urgent need to improve the safety and quality of perioperative care in developing countries and integrate perioperative care into global health efforts. (Anesth Analg 2019;128:1286-91)

\section{KEY POINTS \\ - Question: What is the intraoperative mortality in a Central Referral Hospital of a low-income country? \\ - Findings: Overall intraoperative mortality was 76 (95\% confidence interval [CI], 44-124) per 100,000 surgeries. It ranged from 57 deaths per 100,000 surgeries (95\% Cl, 26-108) in the early cohort to 133 deaths per 100,000 surgeries (95\% Cl, 56-286) in the late cohort. \\ - Meaning: Intraoperative mortality is high in Malawi, which highlights the need for increased investments in safe surgical and anesthetic care in low-income countries.}

S urgery is an essential component of global health, but many low- and middle-income countries struggle to provide timely access to safe surgical and anesthetic care. Sub-Saharan Africa in particular has a disproportionate share of the global burden of surgical disease, and many patients with potentially reversible surgical conditions in this region do not have access to definitive care. ${ }^{1}$ Surgical care occurs over a continuum, and patients require highquality preoperative, intraoperative, and postoperative

From the *Department of Anesthesiology and Critical Care, Columbia University College of Physicians and Surgeons, New York, New York; †Department of Biostatistics, Icahn School of Medicine at Mt Sinai, New York, New York; $\ddagger$ Department of Anesthesiology and Pediatrics, University of North Carolina at Chapel Hill, Chapel Hill, North Carolina; §Department of Anesthesiology, Kamuzu Central Hospital, Lilongwe, Malawi; ॥Department of Anesthesiology, Columbia University College of Physicians and Surgeons, New York, New York; \Department of Epidemiology, Columbia University Mailman School of Public Health, New York, New York; and \#Department of Surgery, University of North Carolina at Chapel Hill, Chapel Hill, North Carolina. attention. Although it is likely that many developing health care systems struggle in all these realms, data describing the separate components of the surgical care continuum are scarce. The purpose of this study was to measure the intraoperative mortality for patients undergoing surgery in a Central Referral Hospital of Malawi. As a secondary aim, we sought to compare intraoperative mortality between 2 time periods, accounting for demographics and surgical type. As a third aim, we explored the factors associated with

Accepted for publication July 16, 2018.

Funding: This study was supported in part by the National Institutes of Health Fogarty International Center Postdoctoral Research Fellowship to Dr Prin.

The authors declare no conflicts of interest.

The contents of the manuscript are solely the responsibility of the authors and do not necessarily reflect the official views of the funding agencies.

Reprints will not be available from the authors.

Address correspondence to Meghan Prin, MD, MS, Department of Anesthesiology and Critical Care, Columbia University Medical Center, 622 W 168th St, PH-505, New York, NY 10032. Address e-mail to prin@bcm.edu. 
intraoperative mortality in both time periods. These data will serve as a benchmark for surgical care improvements at this site and other similar low-resource settings.

\section{METHODS}

This was a retrospective cohort study of patients undergoing surgery at Kamuzu Central Hospital (KCH) in Lilongwe, Malawi. This study was approved by the National Health Sciences Research Council of Malawi and the institutional review boards of Columbia University and the University of North Carolina. The requirement for written informed consent was waived by all. Malawi is a country in Southern Africa with a population of 18 million people, a life expectancy of 63.8 years, and a Human Development Index rank of 170 of 187 countries. $^{2}$ It is the third poorest country in Africa, and its health care system serves as a proxy for lowincome countries in the region. $\mathrm{KCH}$ is a Central Referral Hospital in the central region of Malawi with a catchment area of approximately 5 million.

The data were collected prospectively by clinical officers in anesthesiology at $\mathrm{KCH}$ and maintained in a deidentified computerized database. Data describing daily consecutive cases in the main operating theaters were available for 2 time periods: April 2004 to December 2006 (early cohort, 33 months) and May 2015 to December 2016 (late cohort, 17 months). Data from other time periods were not available because of staffing shortages and infrastructural obstacles. Variables collected included gender, age, American Society of Anesthesiology physical status (ASA-PS) score, surgical procedure, emergency status, anesthetic management, airway management, blood products administered, and postoperative disposition. Surgical procedure was categorized as general surgery (primarily exploratory laparotomies for suspected abdominal pathology), obstetrics and gynecology (inclusive of cesarean deliveries), orthopedics, and all other cases (including urology, otorhinolaryngology, burns, and wound debridements). All data were collected as categorical variables (eg, age was recorded as $<1,1-5,6-14,15-30$, $31-40,41-60$, and $>60$ years). We categorized the ASA-PS score into status I and II versus status III and IV to account for low cell counts.

Missing data accounted for $<3 \%$ of data for all variables except the ASA-PS score, which had $17.5 \%$ missing data and is a potential source of bias. On cases of missing ASA-PS score, we applied multiple imputation by fully conditional specification $^{3}$ based on age, sex, surgical category, and procedure duration. This method assumes that the data are at least missing at random, and we suspect that the pattern of missingness may be related to other variables. For instance, $10.3 \%$ of ASA-PS scores were missing from orthopedic surgery cases compared to $27.6 \%$ missing among other surgical cases (ie, urology, burns, etc). For patients 31-60 years of age, $8.8 \%$ had missing ASA-PS scores, while for patients 15-30 years of age, $22.9 \%$ had missing ASA-PS scores. We used multiple imputation as an attempt to reduce the bias due to missing data, but our estimate of the association between time and mortality could still be biased if missingness depends on the missing variable (ie, missing not at random).

The primary outcome was intraoperative mortality, measured as the number of patients dying during surgery in the operating theater from the time of anesthesia induction to the completion of the procedure. For the observed sample, we first reported the number of patients and the proportion within each time period. Comparisons between cohorts were performed using $\chi^{2}$ tests, and standardized differences were reported between groups. Given the imbalance between the 2 cohorts, propensity scores were obtained to account for potential confounding by observed demographics and surgical factors. A propensity score was calculated to illustrate the probability of a patient being in the late cohort given an observed set of variables: age, sex, emergency status, department, and anesthesia method. Using the propensity score, we then derived the inverse probability of treatment weight (IPTW) for each patient to reduce the imbalance between the 2 time periods. After accounting for IPTW, cohort differences were evaluated by the IPTW proportions and standardized differences. All covariates had standardized differences $<0.10$, and the distribution of propensity scores before and after IPTW is illustrated in Figure 1. We used a logistic regression model to evaluate the association between time period and intraoperative mortality with IPTW adjustment. For comparison, multivariable logistic regression models were performed on the observed and imputed sample to assess the association between time and intraoperative mortality, adjusting for sex, age, ASA-PS
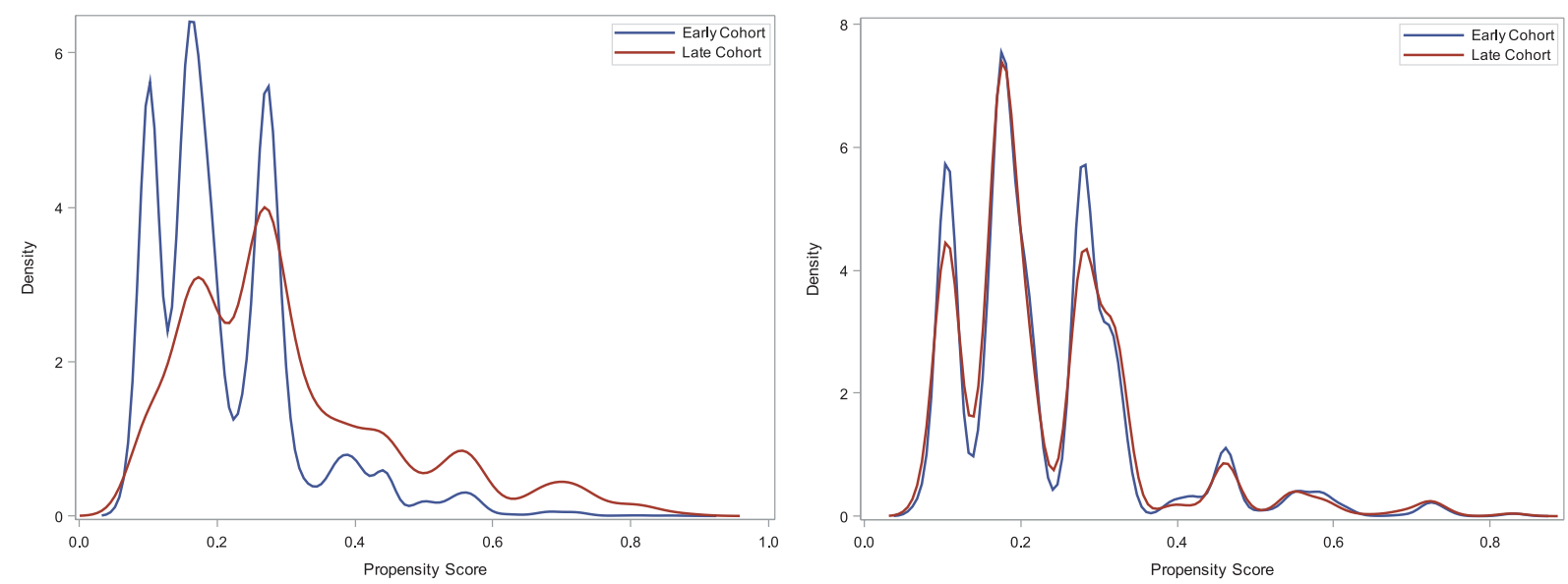

Figure 1. Distribution of propensity scores before and after IPTW. IPTW indicates inverse probability treatment weighting. 


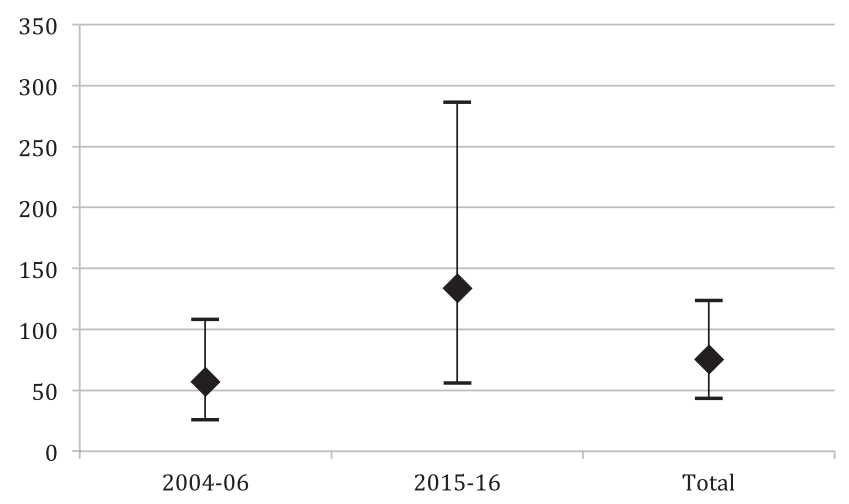

Figure 2. Intraoperative mortality per 100,000 surgeries with $95 \%$ confidence intervals at Kamuzu Central Hospital, Lilongwe, Malawi, in the early and late cohorts, and overall.

score, emergency status, surgical department (eg, general surgery, obstetrics and gynecology, orthopedics), and anesthesia method. Covariates selected for inclusion in the model were based on clinical relevance.

For our third aim, to evaluate whether the demographic and surgical covariates remained consistent in their association with intraoperative mortality, we performed covariate by time interaction with intraoperative mortality as the outcome. There were no significant interactions for any of the covariates; as a result, we pooled the cohort to explore demographic and clinical factors associated with intraoperative mortality. Statistical analyses were conducted using SAS Version 9.4 (SAS Institute, Inc, Cary, NC). A 2-tailed $P$ value of $<.05$ was used to define statistical significance.

\section{RESULTS}

There were a total of 21,090 surgeries performed during the 2 time periods, with 15,846 (75\%) completed in the 33-month early cohort and 5244 (25\%) in the 17-month late cohort. The intraoperative mortality rate in the early cohort was 57 (95\% confidence interval [CI], 26-108) intraoperative deaths per 100,000 surgeries and in the late cohort 133 (95\% CI, 56-286) intraoperative deaths per 100,000 surgeries, with 76 (95\% CI, 44-124) per 100,000 surgeries overall (Figure 2). The cohorts differed in their demographics and case mix. There was a larger proportion of males (33\% vs $28 \%$; $P<.001)$, more emergency status surgeries (53\% vs $43 \%$; $P<.001)$, and more orthopedic cases $(14 \%$ vs $3 \% ; P<.001)$ in the late cohort compared to the early cohort. After IPTW adjustment, we observed that the early and late cohorts were more balanced across the baseline demographics and clinical characteristics (Table 1).

There was no association between intraoperative mortality and the 2 time periods after adjusting for confounding using the IPTW method (odds ratio [OR], 1.6; 95\% CI, $1-2.8 ; P=.08)$. Correspondingly, no association was found between intraoperative mortality and the 2 time periods after adjusting for potential confounding variables in the observed or imputed samples (OR, 2.0; 95\% CI, 0.7-5.4; $P=.17$; and OR, 1.3; 95\% CI, 0.5-3.2; $P=.56$ ) (Table 2).

For exploratory purposes, no interactions were detected between each demographic and clinical factor with time period in their relationship with mortality (all $P \geq .20$ ). After pooling the 2 cohorts, the adjusted analysis found that
ASA-PS score (III or IV versus I or II) and emergency status were significantly associated with intraoperative mortality (imputed sample: OR, 4.4; 95\% CI, 1.5-12.5; $P=.006$; and OR, 7.7; 95\% CI, 2.5-23.6; $P<.001$, respectively). This finding was consistent with the model utilizing the observed sample (Table 3).

\section{DISCUSSION}

This study describes the case mix and intraoperative mortality rate for patients undergoing surgery at a Central Referral Hospital of Malawi. Overall, in combining the early and late cohorts, we found that intraoperative mortality in the study hospital is high (76 deaths per 100,000 surgeries) and did not change between the 2 time periods. Despite the inaccessibility of data between 2007 and 2014 to model the relationship over time, the high overall intraoperative mortality suggests a substantial need for improvements in surgical and anesthetic care in this region.

With increasing recognition of the role of surgery in improving population health, ${ }^{4}$ several metrics have been proposed to monitor and evaluate health care systems and processes of care delivery, ${ }^{5-7}$ but these metrics have rarely been applied in Sub-Saharan Africa. These metrics are also broad in scope and do not necessarily indicate which specific parts of the health care system should be targeted to increase access to quality care (eg, health care providers, infrastructure, anesthesia services). The perioperative mortality rate (POMR) is likely the most generalizable metric for use in low- and middle-income countries because it captures the continuum of surgical care and is applicable to patients of all ages undergoing all types of surgical procedures. However, it can be manipulated by the use of different denominators or time points for survival (eg, 24-hour vs 7-day mortality), ${ }^{8}$ and it requires reliable follow-up by clinical staff or research personnel, which may not be feasible in low-income or low-resource settings.

In areas of Sub-Saharan Africa with maturing surgical and anesthesia delivery systems, more precise details about surgical and anesthetic care, stratified by the preoperative, intraoperative, and postoperative periods, may more efficiently direct quality improvement efforts. Intraoperative mortality serves this purpose, in part, by reflecting the preoperative and intraoperative care. It is also easily measured without postoperative follow-up, making it more feasible for low-resource settings.

The overall intraoperative mortality of 76 (95\% CI, 44 124) per 100,000 surgeries at this study site is higher than recent reports describing the POMR from Rwanda and Kenya. ${ }^{9,10}$ It is important to understand that intraoperative mortality is an underestimation of overall perioperative mortality, and this suggests an even higher POMR for Malawi. While the precise differences between surgical care in Malawi and Rwanda or Kenya are beyond the scope of this article, it is well known that in the past 2 decades since the Rwandan genocide, Rwanda has received foreign investments for the development of quality health care that are not likely generalizable to the region. The study from Kenya was also conducted at a hospital that has had substantial external investments over the past decade. Our study, although limited by the absence of cause-of-death 


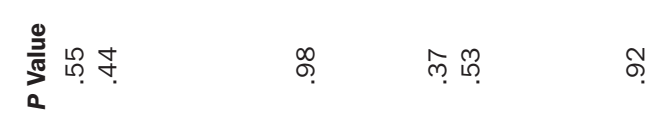

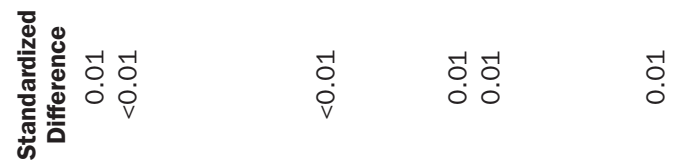

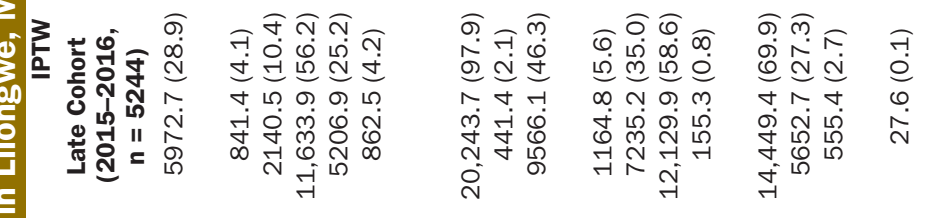

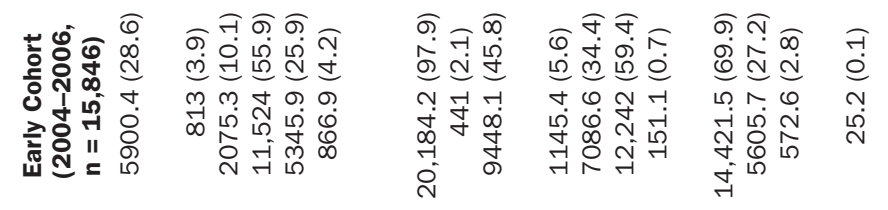

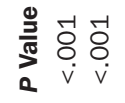
id $\quad$ i
ì

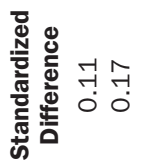

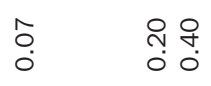

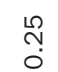

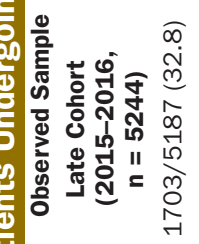

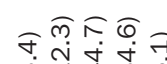

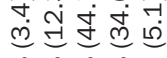

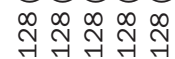

जे चैं

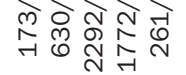

으요

बने

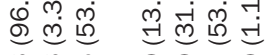

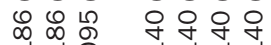

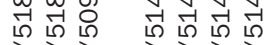

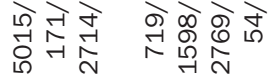

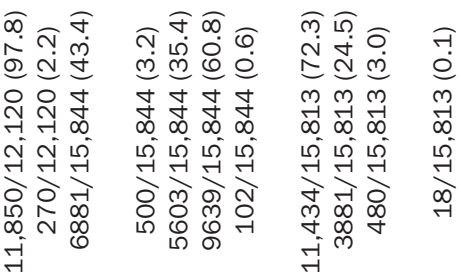

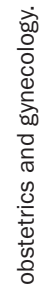

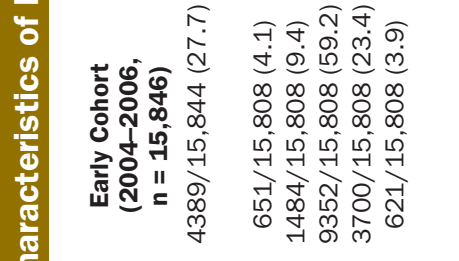

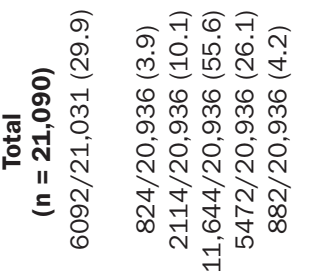

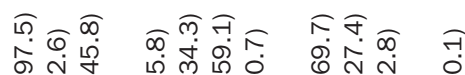

造

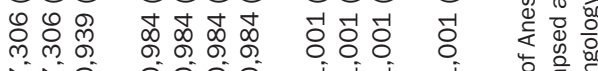
ทำ จำกำ

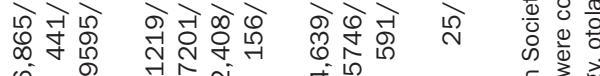

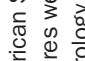




\section{Analysis Type}

Multivariable logistic modela (observed)

Multivariable logistic modela (imputed)

Inverse probability treatment weight logistic model (imputed)

\begin{tabular}{cc}
\multicolumn{2}{c}{ No. Patients (\%) } \\
Early Cohort & Late Cohort \\
$\mathbf{( 2 0 0 4 - 2 0 0 6 )}$ & $\mathbf{( 2 0 1 5 - 2 0 1 6 )}$ \\
$11,636 / 16,317(71.3)$ & $4681 / 16,317(28.7)$ \\
$15,183 / 19,748(76.9)$ & $4565 / 19,748(23.1)$ \\
$15,777 / 20,495(77.0)$ & $4718 / 20,495(23.0)$
\end{tabular}

$\begin{array}{cc}\begin{array}{c}\text { Odds Ratio } \\ \text { (95\% CI) }\end{array} & \boldsymbol{P} \text { Value } \\ 2.0(0.7-5.4) & .17 \\ 1.3(0.5-3.2) & .56 \\ 1.6(0.9-2.8) & .08\end{array}$

Abbreviation: $\mathrm{Cl}$, confidence interval.

aTime period is adjusted for sex, age, American Society of Anesthesiology physical status score, emergency status, surgical department, and anesthesia method.

\begin{tabular}{|c|c|c|c|c|}
\hline \multirow[b]{2}{*}{ Variables } & \multicolumn{2}{|c|}{ Observed Sample } & \multicolumn{2}{|c|}{ Imputed Sample } \\
\hline & $\begin{array}{l}\text { Adjusted OR } \\
(95 \% \mathrm{CI})\end{array}$ & $P$ Value & $\begin{array}{c}\text { Adjusted OR } \\
(95 \% \mathrm{CI})\end{array}$ & $P$ Value \\
\hline Male & $0.9(0.2-4.7)$ & .95 & $1.0(0.3-3.2)$ & $>.99$ \\
\hline Age $(y)$ & & .91 & & .58 \\
\hline$<1$ & $2.0(0.2-20.6)$ & .56 & $3.1(0.7-13.8)$ & .14 \\
\hline $1-14$ & $0.6(0.1-6.2)$ & .69 & $1.1(0.3-4.9)$ & .87 \\
\hline $15-30$ & Reference & & Reference & \\
\hline $31-60$ & $1.0(0.3-3.3)$ & .97 & $0.9(0.3-2.8)$ & .94 \\
\hline$>60$ & $1.9(0.2-19.2)$ & .57 & $1.1(0.1-9.8)$ & .92 \\
\hline ASA-PS score & & $<.001$ & & .006 \\
\hline | or || & Reference & & Reference & \\
\hline III or IV & 7.9 (2.5-24.9) & & $4.4(1.5-12.5)$ & \\
\hline Emergency status & $5.2(1.4-19.3)$ & .01 & $7.7(2.5-23.6)$ & $<.001$ \\
\hline Department ${ }^{a}$ & & .77 & & .79 \\
\hline OBGyn & Reference & & Reference & \\
\hline General & $1.5(0.1-18.2)$ & .75 & $1.5(0.1-14.9)$ & .75 \\
\hline Orthopedics & $0.7(0.1-4.0)$ & .69 & $1.6(0.4-6.3)$ & .49 \\
\hline Anesthesia method ${ }^{b}$ & & .06 & & .03 \\
\hline Spinal & Reference & & Reference & \\
\hline General & $0.1(0.0-1.1)$ & & $0.1(0.0-0.8)$ & \\
\hline
\end{tabular}

Abbreviations: ASA-PS, American Society of Anesthesiology physical status; Cl, confidence interval; OBGyn, obstetrics and gynecology; OR, odds ratio. asurgical procedures in "other" departments were excluded from the model due to low cell counts.

bSaddle, peripheral, or local block, and other or unknown anesthesia methods were excluded from the model due to low cell counts.

data or a true POMR, provides an important benchmark for efforts to improve surgery in Malawi, and it may be more generalizable to regions where significant investments in surgical care delivery have not yet been made.

Factors associated with intraoperative death at this study site included ASA-PS score of III or IV and emergency status. While these factors are well-known predictors of perioperative mortality in high-income regions, ${ }^{11}$ there is a paucity of data describing perioperative mortality in Sub-Saharan Africa, and this study is one of the largest of its kind to provide clinical data confirming these associations. While the association of emergency status with intraoperative death is not unexpected, 9,12 the OR for intraoperative mortality with emergency surgery in this study (imputed sample adjusted OR, 7.7; 95\% CI, 2.5-23.6) is higher than that found in both high-income settings ${ }^{13}$ and in recent data from Kenya. ${ }^{10}$ The proportion of emergent surgeries at this site $(46 \%)$ is striking and may be due to several factors. Given the increase in the unweighted proportion of male patients, orthopedic cases, and patients with ASA-PS status III or IV, this may be attributable to an increased burden of traumatic injuries over time. Alternatively, elective case cancellation is common in this region because of infrastructural limitations, ${ }^{14-16}$ and this may be associated with the volume of emergency surgery. Increased efforts at surgical care rollout in this region may have also led to more patients seeking surgery, but staffing and equipment may not have risen to meet the demand. Finally, in 2011, the financial aid to Malawi from the United Kingdom decreased dramatically, which may have affected the health care budget and available services. ${ }^{17}$ Surgical volume at this site increased only marginally over the study period and does not necessarily explain a high emergency case volume. Identifying the precipitating factors for emergency surgery and improving access to timely surgical care may be an important first step toward improving surgical safety in this region.

Improvements in quality surgical and anesthetic care in Malawi, and the region as a whole, will have to address infrastructural limitations. Although this study was performed at a Central Referral Hospital with some of the best staffing and resources in the country, the practice of anesthesia and surgery in Malawi is different than the standard in high-income settings. Anesthetic equipment such as ventilators, supplemental oxygen, end-tidal carbon dioxide monitoring or capnography, and warming devices is routinely absent. Available equipment is often old and cannot provide more modern interventions such as positive end-expiratory pressure or a titratable fraction of inspired oxygen. Medications used daily in high-income 
countries (eg, propofol, phenylephrine, fentanyl) are not routinely available. Outside the central hospital level, equipment and medications are even more scarce. For example, although every district hospital provides general anesthesia, only about half of hospitals in Malawi have a functioning anesthesia machine. ${ }^{18}$ Thus, the intraoperative mortality rate reported in this study likely represents the low range for intraoperative mortality nationwide and cannot reasonably be expected to improve without addressing these limitations.

Improvements will also have to take into account the differences in health care provider structure. Nonphysician clinical officers provide $>90 \%$ of surgery and anesthetic care in Malawi. ${ }^{18}$ The current training for specialty clinical officers in anesthesiology or surgery is an 18-month course, after which the officers independently p rovide a nesthesia, surgery, and critical care. This strategy has allowed the country to develop a cadre of trained health care providers while avoiding brain drain, but this training alone cannot prepare officers to manage all intraoperative complications. The high intraoperative mortality may indicate the need for improved education to address higher acuity patients and emergency cases. This may include simulation training and continuing education programs developed especially for clinical officers. Recruitment and retention of consultant physicians trained in anesthesia and surgery for oversight of the clinical officer workforce are other strategies to improve nationwide care.

This study has several limitations. First, because this was a secondary analysis of an existing database, we could not account for readmissions or repeat operations, which would reasonably be expected to be higher risk than initial procedures. Second, we did not have more granular data describing the operative interventions, intraoperative vital signs and events, or the proximate cause of death. Therefore, we could not assess the specific treatment modalities that may prevent further intraoperative events. The ASA-PS data may also be a potential source of bias because the pattern of its missingness may have been related to other variables such as surgical type or patient age. Finally, the daily case data from 2007 to 2014 were unavailable, so we were unable to assess trends in intraoperative mortality over that time period.

In summary, intraoperative mortality is an easily measurable indicator for surgical care quality in low-resource settings that focuses attention on a critically important time period for improving patient safety. Intraoperative mortality is high in Malawi and is not decreasing. This may be attributable to many factors, including a higher proportion of emergency surgery and/or changes in case mix. Efforts at improving safe surgery should focus on decreasing the need for emergency surgical care.

\section{DISCLOSURES}

Name: Meghan Prin, MD, MS.

Contribution: This author helped to conceive and design the project, to perform data analysis, to write the manuscript, and had full access to all the data in the study.

Name: Stephanie Pan, MS.

Contribution: This author helped with statistical analysis and manuscript preparation.

Name: Janey Phelps, MD.

Contribution: This author helped to collect data and to write the manuscript.

Name: Godfrey Phiri, CO.
Contribution: This author helped to collect the data and to write the manuscript.

Name: Guohua Li, MD, DrPH.

Contribution: This author helped with the statistical analyses and writing the manuscript.

Name: Anthony Charles, MD, MPH.

Contribution: This author helped to conceive and design the project, and to write the manuscript.

This manuscript was handled by: Angela Enright, MB, FRCPC.

\section{REFERENCES}

1. Debas HT, Gosselin R, McCord C, Thind A. Surgery. In: Jamison DT, Breman JG, Measham AR, et al, eds. Disease Control Priorities in Developing Countries. 2nd ed. Washington, DC: Oxford University Press; 2006:1-26.

2. UN: Human Development Reports. United Nations Development Programme. Human Development Indicators. 2016. Available at: http://hdr.undp.org/en/countries/profiles/MWI. Accessed December 28, 2017.

3. Liu Y, De A. Multiple imputation by fully conditional specification for dealing with missing data in a large epidemiologic study. Int J Stat Med Res. 2015;4:287-295.

4. Ozgediz D, Riviello R. The "other" neglected diseases in global public health: surgical conditions in Sub-Saharan Africa. PLoS Med. 2008;5:e121.

5. Watters DA, Hollands MJ, Gruen RL, et al. Perioperative mortality rate (POMR): a global indicator of access to safe surgery and anaesthesia. World J Surg. 2015;39:856-864.

6. Hughes CD, McClain CD, Hagander L, et al. Ratio of cesarean deliveries to total operations and surgeon nationality are potential proxies for surgical capacity in central Haiti. World J Surg. 2013;37:1526-1529.

7. Samuel JC, Tyson AF, Mabedi C, et al. Development of a ratio of emergent to total hernia repairs as a surgical capacity metric. Int J Surg. 2014;12:906-911.

8. Ariyaratnam R, Palmqvist CL, Hider P, et al. Toward a standard approach to measurement and reporting of perioperative mortality rate as a global indicator for surgery. Surgery. 2015;158:17-26.

9. Rickard JL, Ntakiyiruta G, Chu KM. Associations with perioperative mortality rate at a major referral hospital in Rwanda. World J Surg. 2016;40:784-790.

10. Sileshi B, Newton MW, Kiptanui J, et al. Monitoring anesthesia care delivery and perioperative mortality in Kenya utilizing a provider-driven novel data collection tool. Anesthesiology. 2017;127:250-271.

11. Koo CY, Hyder JA, Wanderer JP, Eikermann M, Ramachandran SK. A meta-analysis of the predictive accuracy of postoperative mortality using the American Society of Anesthesiologists' physical status classification system. World J Surg. 2015;39:88-103.

12. Glance LG, Lustik SJ, Hannan EL, et al. The surgical mortality probability model: derivation and validation of a simple risk prediction rule for noncardiac surgery. Ann Surg. 2012;255:696-702.

13. Whitlock EL, Feiner JR, Chen LL. Perioperative mortality, 2010 to 2014: a retrospective cohort study using the National Anesthesia Clinical Outcomes Registry. Anesthesiology. 2015;123:1312-1321.

14. Chalya PL, Gilyoma JM, Mabula JB, et al. Incidence, causes and pattern of cancellation of elective surgical operations in a university teaching hospital in the Lake Zone, Tanzania. Afr Health Sci. 2011;11:438-443.

15. Bhuiyan MM, Mavhungu R, Machowski A. Provision of an emergency theatre in tertiary hospitals is cost-effective: audit and cost of cancelled planned elective general surgical operations at Pietersburg Hospital, Limpopo Province, South Africa. S Afr Med J. 2017;107:239-242.

16. Prin M, Eaton J, Mtalimanja O, Charles A. High elective surgery cancellation rate in Malawi primarily due to infrastructural limitations. World J Surg. 2018;42:1597-1602.

17. Tran M. Britain suspends aid to Malawi. The Guardian. London, UK: Guardian Media Group; 2011.

18. Henry JA, Frenkel E, Borgstein E, Mkandawire N, Goddia C. Surgical and anaesthetic capacity of hospitals in Malawi: key insights. Health Policy Plan. 2015;30:985-994. 